GLASNIK MATEMATIČKI

Vol. 40(60)(2005), $121-132$

\title{
DELAY DEPENDENT STABILITY CRITERION FOR TIME DISCRETE LINEAR SYSTEMS
}

\author{
X.H. TANG And S.S. Cheng \\ Central South University, China and Tsing Hua University, Taiwan
}

\begin{abstract}
It is shown that every solution of the linear difference system with constant coefficients and delays tends to zero if a certain matrix derived from the coefficient matrix is a M-matrix and the diagonal delays satisfy delay dependent conditions.
\end{abstract}

\section{INTRODUCTION}

Delayed linear difference systems with constant coefficients of the form

$$
\Delta x_{i}(n)=-\sum_{j=1}^{m} a_{i j} x_{j}\left(n-k_{i j}\right), i=1,2, \ldots, m,
$$

with

$$
k_{i j} \in\{0,1,2, \ldots\}, 1 \leq i, j \leq m \text { and } a_{i i}>0, i=1,2, \ldots, m,
$$

arise in many mathematical models involving interacting variables. As a specific example, consider the following dynamical model of a two-nation arms race. Let $A(n)$ and $B(n)$ be the armament expenditures of two countries $A$ and $B$ in year $n$. The increase $A(n+1)-A(n)$ in expenditures by $A$ in two consecutive years usually depends on the expenditures of $A$ and $B$ in previous years. If we assume that large expenditures in the $(n-\tau)$-th year will deplete a country's treasury in the $n$-th year, it is reasonable that

$$
A(n+1)-A(n)=-\alpha A(n-\tau)+\beta B(n-\sigma),
$$

2000 Mathematics Subject Classification. 39A10, 39A11.

Key words and phrases. M-matrix, delay, stability.

Supported by NNSF of China. 
where $\alpha$ is a positive proportionality constant, and $\beta$ is a coefficient saying to which degree the country A does not distrust the country $B$. Similar assumptions for country $B$ lead to

$$
B(n+1)-B(n)=-\gamma B(n-\xi)+\delta A(n-\zeta),
$$

where $\gamma>0$. A natural question is whether the expenditures $A(n)$ and $B(n)$ will tend to zero since this situation corresponds to ultimate disarmament. In mathematical terms, we are concerned with the question as to whether (1) is asymptotically stable (i.e., every solution of (1) tends to zero).

When each $k_{i j}$ is zero, it is well known that system (1) is asymptotically stable if, and only if, the spectral radius of the matrix $I-A$ is strictly less than 1 , where $I$ is an identity matrix and $A=\left(a_{i j}\right)$.

When some $k_{i j}$ is not zero, it is well known that (1) can be embedded into a system of the form

$$
y(n+1)=B y(n) .
$$

Then the asymptotic stability of (1) is determined from evaluating the spectral radius of the matrix $B$. Although numerical techniques can be utilized to calculate the spectral radius of $B$, it is of great interest to determine explicit conditions which guarantee the asymptotic stability of (1). This is particularly true when (1) is viewed as the first approximation of a nonlinear model.

In the case when (1) is of the form

$$
\begin{aligned}
& x_{1}(n+1)-x_{1}(n)+a x_{1}(n-k)+b x_{2}(n-k)=0, \\
& x_{2}(n+1)-x_{2}(n)+c x_{1}(n-k)+d x_{2}(n-k)=0,
\end{aligned}
$$

a necessary and sufficient condition for asymptotic stability is known [1]. In particular, when $c=0$, a necessary and sufficient condition for the above two variable constant delay system to be asymptotically stable is that

$$
0<a, d<2 \cos \frac{k \pi}{2 k+1}
$$

and when

$$
\left(\begin{array}{ll}
a & b \\
c & d
\end{array}\right)=q\left(\begin{array}{cc}
\cos t & -\sin t \\
\sin t & \cos t
\end{array}\right), q \in R,|t| \leq \frac{\pi}{2},
$$

a necessary and sufficient condition is

$$
0<q<2 \cos \frac{k \pi+|t|}{2 k+1} .
$$

For the general case, it can be shown that when the spectral radius of the matrix $\left(\delta_{i j}-a_{i j}\right)_{m \times m}$, where $\delta_{i j}$ is the Kronecker delta, is less than one, then (1) is asymptotically stable [2]. Therefore, explicit sufficient condition can be constructed by demanding a natrural norm of $\left(\delta_{i j}-a_{i j}\right)$ to be less than 1. Such a condition, however, is independent of the delays $k_{i j}$. On the 
other hand, (4) and (5) are delay dependent conditions. Therefore, sufficient conditions for (1) should be expected.

In this paper, we will give a sufficient condition which guarantees the asymptotic stability of (1) and which involves the delays $k_{i i}, i=1, \ldots, m$. For convenience, we recall the concept of a $M$-matrix (see e.g. Fiedler [3]): A $n \times n$ matrix $C=\left(c_{i j}\right)$ is an $M$-matrix if $c_{i j} \leq 0$ for $i \neq j$, and all principal minors of $C$ are positive. There are many equivalent formulations of this concept (see e.g. Fiedler [3, Theorem 5.1.]). In particular, if $C$ is an $M$-matrix, then $C^{-1}$ is a positive matrix.

\section{Stability CRiterion}

To the $n \times n$ matrix $A$, we associate a new matrix $\tilde{A}=\left(\tilde{a}_{i j}\right)$ defined by

$$
\tilde{a}_{i i}=a_{i i}
$$

for $i=1,2, \ldots, m$, and

$$
\tilde{a}_{i j}=-\frac{\left(3 k_{i i}+4\right)^{2}+\left(k_{i i}+1\right) a_{i i}\left[\left(k_{i i}+2\right)\left(3 k_{i i}+4\right)+2 k_{i i}\left(k_{i i}+1\right)^{2} a_{i i}\right]}{\left(3 k_{i i}+4\right)^{2}-\left(k_{i i}+1\right) a_{i i}\left[\left(k_{i i}+2\right)\left(3 k_{i i}+4\right)+2 k_{i i}\left(k_{i i}+1\right)^{2} a_{i i}\right]}\left|a_{i j}\right|,
$$

for $i \neq j$ and $i, j=1,2, \ldots, m$.

Theorem 2.1. Assume that

$$
a_{i i}<\frac{3}{2\left(k_{i i}+1\right)}+\frac{1}{2\left(k_{i i}+1\right)^{2}}, i=1,2, \ldots, m .
$$

If $\tilde{A}$ is an $M$-matrix, then every solution $\left(x_{1}(n), x_{2}(n), \ldots, x_{m}(n)\right)$ of (1) tends to 0 as $n \rightarrow \infty$.

We first derive a preparatory result.

Lemma 2.2. Under the conditions of Theorem 2.1, every solution of (1) is bounded.

Proof. Assume to the contrary that $\left(x_{1}(n), x_{2}(n), \ldots, x_{m}(n)\right)$ is an unbounded solution of (1). Without loss of generality, we may assume that

$$
\limsup _{n \rightarrow \infty}\left|x_{i}(n)\right|=\infty, i=1,2, \ldots, k(\leq m),
$$

and

$$
\left|x_{i}(n)\right| \leq M, \text { for } n \geq 0 ; i=k+1, k+2, \ldots, m .
$$

Let $N$ be the smallest integer such that $N>k_{i i}$ for all $i$. There is an integer $N_{1}>N$ such that for each $i=1,2, \ldots, k$, the maximum of the sequence $\left\{\left|x_{i}(n)\right|\right\}$ in set $\left\{0,1, \ldots, N_{1}\right\}$ is attained at a point in the set $\{N, N+$ $\left.1, \ldots, N_{1}\right\}$. Fix $i=1,2, \ldots, k$. For each integer $l \geq 1$, let $n_{i l} \in\{N, N+$ $\left.1, \ldots, N_{1}+l\right\}$ be such that $\left|x_{i}\left(n_{i l}\right)\right|=\max \left\{\left|x_{i}(n)\right|: 0 \leq n \leq N_{1}+l\right\}$. We may 
assume that $\left\{n_{i l}\right\}_{l=1}^{\infty}$ is a nondecreasing sequence. By taking the subsequences if necessary, we have $k$ sequences $\left\{n_{i l}\right\}_{l=1}^{\infty}$ of integers, $i=1,2, \ldots, k$, such that

$$
n_{i l} \uparrow \infty,\left|x_{i}\left(n_{i l}\right)\right| \uparrow \infty \text { as } l \rightarrow \infty,\left|x_{i}(n)\right| \leq\left|x_{i}\left(n_{i l}\right)\right| \text { for } 0 \leq n \leq n_{l},
$$

for $i=1,2, \ldots, k$, where $n_{l}=\max \left\{n_{i l}: i=1,2, \ldots, k\right\}$. Again by taking the subsequences if necessary, we may assume for each $i=1,2, \ldots, k$, all the terms in the sequence $\left\{x_{i}\left(n_{i l}\right)\right\}_{l=1}^{\infty}$ are of the same sign. Without loss of generality (i.e. by using $-x_{i}(n)$ instead of $x_{i}(n)$ and $-a_{i j}$ instead of $a_{i j}$ for $j \neq i$, if necessary), we may assume that $\left|x_{i}\left(n_{i l}\right)\right|=x_{i}\left(n_{i l}\right)$. Then

$$
\left|x_{i}(n)\right| \leq x_{i}\left(n_{i l}\right), 0 \leq n<n_{l}, i=1,2, \ldots, k .
$$

It follows from (1) that

$$
\begin{aligned}
0 \leq & -\sum_{j=1}^{m} a_{i j} x_{j}\left(n_{i l}-k_{i j}-1\right) \leq-a_{i i} x_{i}\left(n_{i l}-k_{i i}-1\right) \\
& +\sum_{j \neq i}^{k}\left|a_{i j}\right| x_{j}\left(n_{j l}\right)+M \sum_{j=k+1}^{m}\left|a_{i j}\right|,
\end{aligned}
$$

or

(11) $x_{i}\left(n_{i l}-k_{i i}-1\right) \leq \frac{1}{a_{i i}}\left[\sum_{j \neq i}^{k}\left|a_{i j}\right| x_{j}\left(n_{j l}\right)+M \sum_{j=k+1}^{m}\left|a_{i j}\right|\right], i=1,2, \ldots, k$.

Set

$$
\alpha_{i l}=\frac{1}{a_{i i}}\left[\sum_{j \neq i}^{k}\left|a_{i j}\right| x_{j}\left(n_{j l}\right)+M \sum_{j=k+1}^{m}\left|a_{i j}\right|\right], i=1,2, \ldots, k .
$$

We will now show

$$
a_{i i} x_{i}\left(n_{i l}\right)+\sum_{j \neq i}^{k} \tilde{a}_{i j} x_{j}\left(n_{j l}\right) \leq M \sum_{j=k+1}^{m}\left|\tilde{a}_{i j}\right|, i=1,2, \ldots, k .
$$

If $x_{i}\left(n_{i l}\right) \leq \alpha_{i l}$, then (13) obviously holds. If $x_{i}\left(n_{i l}\right)>\alpha_{i l}$, then by (11) and (12) there exists an integer $l_{i}^{*}$ with $0 \leq l_{i}^{*} \leq k_{i i}$ such that

$$
x_{i}\left(n_{i l}-l_{i}^{*}-1\right) \leq \alpha_{i l} \text { and } x_{i}\left(n_{i l}-l_{i}^{*}\right)>\alpha_{i l} .
$$

Let $\xi_{i l} \in(0,1]$ such that

$$
\begin{aligned}
x_{i}\left(n_{i l}\right. & \left.-l_{i}^{*}\right)-\xi_{i l}\left[x_{i}\left(n_{i l}-l_{i}^{*}\right)-x_{i}\left(n_{i l}-l_{i}^{*}-1\right)\right] \\
& =x_{i}\left(n_{i l}-l_{i}^{*}-1\right)+\left(1-\xi_{i l}\right)\left[x_{i}\left(n_{i l}-l_{i}^{*}\right)-x_{i}\left(n_{i l}-l_{i}^{*}-1\right)\right] \\
& =\alpha_{i l} .
\end{aligned}
$$

From (1) we have

$$
\Delta x_{i}(n) \leq a_{i i}\left[-x_{i}\left(n-k_{i i}\right)+\alpha_{i l}\right] \leq a_{i i}\left(\left|x_{i}\left(n_{i l}\right)\right|+\alpha_{i l}\right), N \leq n \leq n_{l} .
$$


For $n_{i l}-l_{i}^{*}-1 \leq n \leq n_{i l}-1$, summing (15) and using (14), we have

$$
\begin{aligned}
\alpha_{i l}-x_{i}\left(n-k_{i i}\right) & \leq \sum_{j=n-k_{i i}}^{n_{i l}-l_{i}^{*}-2} \Delta x_{i}(j)+\left(1-\xi_{i l}\right) \Delta x_{i}\left(n_{i l}-l_{i}^{*}-1\right) \\
& \leq a_{i i}\left(\left|x_{i}\left(n_{i l}\right)\right|+\alpha_{i l}\right)\left(n_{i l}+k_{i i}-l_{i}^{*}-\xi_{i l}-n\right),
\end{aligned}
$$

for $n_{i l}-l_{i}^{*}-1 \leq n \leq n_{i l}-1$. Substituting this into the first inequality in (15), we obtain

$\Delta x_{i}(n) \leq a_{i i}^{2}\left(\left|x_{i}\left(n_{i l}\right)\right|+\alpha_{i l}\right)\left(n_{i l}+k_{i i}-l_{i}^{*}-\xi_{i l}-n\right), n_{i l}-l_{i}^{*}-1 \leq n \leq n_{i l}-1$.

Combining this and (15), we have

$$
\Delta x_{i}(n) \leq a_{i i}\left(\left|x_{i}\left(n_{i l}\right)\right|+\alpha_{i l}\right) \min \left\{1, a_{i i}\left(n_{i l}+k_{i i}-l_{i}^{*}-\xi_{i l}-n\right)\right\},
$$

for $n_{i l}-l_{i}^{*}-1 \leq n \leq n_{i l}-1$. We consider the following two cases:

CASE $1 . l_{i}^{*}+\xi_{i l} \leq 2\left(k_{i i}+1\right)^{2} /\left(3 k_{i i}+4\right)$. In this case, by (16) and (7), we have

$$
\begin{aligned}
& x_{i}\left(n_{i l}\right)-\alpha_{i l} \\
& =\sum_{n=n_{i l}-l_{i}^{*}}^{n_{i l}-1} \Delta x_{i}(n)+\xi_{i l} \Delta x_{i}\left(n_{i l}-l_{i}^{*}-1\right) \\
& \leq a_{i i}^{2}\left(\left|x_{i}\left(n_{i l}\right)\right|+\alpha_{i l}\right)\left[\sum_{n=n_{i l}-l_{i}^{*}}^{n_{i l}-1}\left(n_{i l}+k_{i i}-l_{i}^{*}-\xi_{i l}-n\right)+\xi_{i l}\left(k_{i i}+1-\xi_{i l}\right)\right] \\
& =a_{i i}^{2}\left(\left|x_{i}\left(n_{i l}\right)\right|+\alpha_{i l}\right)\left[\left(k_{i i}+1\right)\left(l_{i}^{*}+\xi_{i l}\right)-\frac{1}{2}\left(l_{i}^{*}+\xi_{i l}\right)^{2}-\frac{1}{2}\left(l_{i}^{*}+\xi_{i l}^{2}\right)\right] \\
& \leq a_{i i}^{2}\left(\left|x_{i}\left(n_{i l}\right)\right|+\alpha_{i l}\right)\left[\left(k_{i i}+1\right)\left(l_{i}^{*}+\xi_{i l}\right)-\frac{k_{i i}+2}{2\left(k_{i i}+1\right)}\left(l_{i}^{*}+\xi_{i l}\right)^{2}\right] \\
& \leq \frac{4\left(k_{i i}+1\right)^{4}}{\left(3 k_{i i}+4\right)^{2}} a_{i i}^{2}\left(\left|x_{i}\left(n_{i l}\right)\right|+\alpha_{i l}\right) \\
& \leq \frac{\left(k_{i i}+1\right)}{\left(3 k_{i i}+4\right)^{2}} a_{i i}\left[\left(k_{i i}+2\right)\left(3 k_{i i}+4\right)+2 k_{i i}\left(k_{i i}+1\right)^{2} a_{i i}\right]\left(\left|x_{i}\left(n_{i l}\right)\right|+\alpha_{i l}\right) .
\end{aligned}
$$

CASE $2 . l_{i}^{*}+\xi_{i l}>2\left(k_{i i}+1\right)^{2} /\left(3 k_{i i}+4\right)$. In this case, there exists an integer $m_{i}^{*}$ and $\eta_{i l} \in[0,1)$ such that

$$
m_{i}^{*}+\eta_{i l}=\frac{2\left(k_{i i}+1\right)^{2}}{3 k_{i i}+4} .
$$


Consequently, from (16) we conclude that

$$
\begin{aligned}
& x_{i}\left(n_{i l}\right)-\alpha_{i l} \\
&=\xi_{i l} \Delta x_{i}\left(n_{i l}-l_{i}^{*}-1\right)+\sum_{n=n_{i l}-l^{*}}^{n_{i l}-m_{i}^{*}-2} \Delta x_{i}(n)+\left(1-\eta_{i l}\right) \Delta x_{i}\left(n_{i l}-m_{i}^{*}-1\right) \\
& \quad+\eta_{i l} \Delta x_{i}\left(n_{i l}-m_{i}^{*}-1\right)+\sum_{n=n_{i l}-m_{i}^{*}}^{n_{i l}-1} \Delta x_{i}(n) \\
& \leq a_{i i}\left(\left|x_{i}\left(n_{i l}\right)\right|+\alpha_{i l}\right)\left[\left(\xi_{i l}+l_{i}^{*}-m^{*}-\eta_{i l}\right)+\eta_{i l} a_{i i}\left(k_{i i}+m_{i}^{*}+1-l_{i}^{*}-\xi_{i l}\right)\right. \\
&\left.\quad+a_{i i} \sum_{n=n_{i l}-m_{i}^{*}}^{n_{i l}-1}\left(n_{i l}+k_{i i}-l_{i}^{*}-\xi_{i l}-n\right)\right] \\
&=a_{i i}\left(\left|x_{i}\left(n_{i l}\right)\right|+\alpha_{i l}\right)\left\{\left(\xi_{i l}+l_{i}^{*}\right)\left[1-a_{i i}\left(m_{i}^{*}+\eta_{i l}\right)\right]\right. \\
&+ {\left.\left[a_{i i}\left(k_{i i}+1\right)-1\right]\left(m_{i}^{*}+\eta_{i l}\right)+\frac{1}{2} a_{i i}\left(m_{i}^{*}+\eta_{i l}\right)^{2}-\frac{1}{2} a_{i i}\left(m_{i}^{*}+\eta_{i l}^{2}\right)\right\} } \\
& \leq a_{i i}\left(\left|x_{i}\left(n_{i l}\right)\right|+\alpha_{i l}\right)\left\{\left(\xi_{i l}+l_{i}^{*}\right)\left[1-a_{i i}\left(m_{i}^{*}+\eta_{i l}\right)\right]\right. \\
&\left.+\left[a_{i i}\left(k_{i i}+1\right)-1\right]\left(m_{i}^{*}+\eta_{i l}\right)+\frac{k_{i i}}{2\left(k_{i i}+1\right)} a_{i i}\left(m_{i}^{*}+\eta_{i l}\right)^{2}\right\} \\
& \leq a_{i i}\left(\left|x_{i}\left(n_{i l}\right)\right|+\alpha_{i l}\right)\left\{\left(k_{i i}+1\right)\left[1-a_{i i}\left(m_{i}^{*}+\eta_{i l}\right)\right]\right. \\
&\left.+\left[a_{i i}\left(k_{i i}+1\right)-1\right]\left(m_{i}^{*}+\eta_{i l}\right)+\frac{k_{i i}}{2\left(k_{i i}+1\right)} a_{i i}\left(m_{i}^{*}+\eta_{i l}\right)^{2}\right\} \\
& \leq a_{i i}\left(\left|x_{i}\left(n_{i l}\right)\right|+\alpha_{i l}\right)\left[k_{i i}+1-\left(m_{i}^{*}+\eta_{i l}\right)+\frac{k_{i i}}{2\left(k_{i i}+1\right)} a_{i i}\left(m_{i}^{*}+\eta_{i l}\right)^{2}\right] \\
&= \frac{\left(k_{i i}+1\right)}{\left(3 k_{i i}+4\right)^{2}} a_{i i}\left[\left(k_{i i}+2\right)\left(3 k_{i i}+4\right)+2 k_{i i}\left(k_{i i}+1\right)^{2} a_{i i}\right]\left(\left|x_{i}\left(n_{i l}\right)\right|+\alpha_{i l}\right) . \\
&=
\end{aligned}
$$

Combining the above two cases, we have

$$
\begin{aligned}
a_{i i} x_{i}\left(n_{i l}\right) \leq & \frac{\left(3 k_{i i}+4\right)^{2}+\left(k_{i i}+1\right) a_{i i}\left[\left(k_{i i}+2\right)\left(3 k_{i i}+4\right)+2 k_{i i}\left(k_{i i}+1\right)^{2} a_{i i}\right]}{\left(3 k_{i i}+4\right)^{2}-\left(k_{i i}+1\right) a_{i i}\left[\left(k_{i i}+2\right)\left(3 k_{i i}+4\right)+2 k_{i i}\left(k_{i i}+1\right)^{2} a_{i i}\right]} \\
& \times\left[\sum_{j \neq i}^{k}\left|a_{i j}\right| x_{j}\left(n_{j l}\right)+M \sum_{j=k+1}^{m}\left|a_{i j}\right|\right]
\end{aligned}
$$

for $i=1,2, \ldots, k$, which implies (13) is true. 
Let $\tilde{A}_{k}=\left(\tilde{a}_{i j}\right)_{k \times k}$ denote the $k$-th leading principal submatrix of $\tilde{A}$. Then $\tilde{A}_{k}$ is also an $M$-matrix of $k$ order, and so $\tilde{A}_{k}^{-1}>0$. Hence, it follows from (13) that

$$
\begin{aligned}
& \left(x_{1}\left(n_{1 l}\right), x_{2}\left(n_{2 l}\right), \ldots, x_{k}\left(n_{k l}\right)\right)^{T} \\
& \leq M \tilde{A}_{k}^{-1}\left(\sum_{j=k+1}^{m}\left|\tilde{a}_{1 j}\right|, \sum_{j=k+1}^{m}\left|\tilde{a}_{2 j}\right|, \cdots, \sum_{j=k+1}^{m}\left|\tilde{a}_{k j}\right|\right)^{T},
\end{aligned}
$$

for $l=1,2, \ldots$. From this, we conclude that

$$
\limsup _{l \rightarrow \infty}\left|x_{i}\left(n_{i l}\right)\right|<\infty, i=1,2, \ldots, k,
$$

which is contrary to the fact that $\left|x_{i}\left(n_{i l}\right)\right| \rightarrow \infty$ as $l \rightarrow \infty, i=1,2, \ldots, k$, and so the proof is complete.

We now turn to the proof of Theorem 2.1. Let $\left(x_{1}(n), x_{2}(n), \ldots, x_{n}(n)\right)$ be a solution of (1) for $n=0,1,2, \ldots$, . We will prove that

$$
\lim _{n \rightarrow \infty} x_{i}(n)=0, i=1,2, \ldots, m
$$

in two possible cases.

CASE 1. $\left\{\sum_{j=1}^{m} a_{i j} x_{j}\left(n-k_{i j}\right)\right\}_{n=0}^{\infty}, i=1,2, \ldots, m$, all are nonoscillatory sequences. Then $\left\{\Delta x_{i}(n)\right\}_{n=0}^{\infty}$ are eventually sign-definite, and so by Lemma 2.2, the limits $c_{i}=\lim _{n \rightarrow \infty} x_{i}(n)$ exist for $i=1,2, \ldots, m$. It follows that $\Delta x_{i}(n) \rightarrow 0$ as $n \rightarrow \infty, i=1,2, \ldots, m$. By (1), we have

$$
\sum_{j=1}^{m} a_{i j} c_{j}=0, i=1,2, \ldots, m
$$

which implies that

$$
a_{i i}\left|c_{i}\right|-\sum_{j \neq i}^{m}\left|a_{i j}\right|\left|c_{j}\right| \leq 0, i=1,2, \ldots, m .
$$

Set $\hat{A}=\left(\hat{a}_{i j}\right)$, where $\hat{a}_{i i}=a_{i i}$ and $\hat{a}_{i j}=-\left|a_{i j}\right|$ for $j \neq i$. Then $\hat{A} \geq \tilde{A}$ and $\hat{A}$ has non-positive off-diagonal entries. In view of [4, Theorem 2.5.4], the matrix $\hat{A}$ is also an $M$-matrix. Since (18) can be expressed as the matrix inequality $\hat{A}\left(\left|c_{1}\right|, \ldots,\left|c_{m}\right|\right)^{T} \leq(0, \ldots, 0)^{T}$, by applying the positive matrix $\hat{A}^{-1}$ to both sides, we conclude that $c_{1}=c_{2}=\cdots=c_{m}=0$.

CASE 2. At least one of the sequences $\left\{\sum_{j=1}^{m} a_{i j} x_{j}\left(n-k_{i j}\right)\right\}_{n=0}^{\infty}, i=$ $1,2, \ldots, m$, is oscillatory. Set

$$
U_{i}=\limsup _{n \rightarrow \infty}\left|x_{i}(n)\right|, i=1,2, \ldots, m .
$$

By Lemma $2.2, U_{i}<\infty, i=1,2, \ldots, m$. It suffices to prove that $U_{1}=$ $U_{2}=\ldots=U_{n}=0$. By rearranging the indices, we may assume that $\left\{\sum_{j=1}^{m} a_{i j} x_{j}\left(n-k_{i j}\right)\right\}_{n=0}^{\infty}, i=1,2, \ldots, k$, are oscillatory and $\left\{\sum_{j=1}^{m} a_{i j} x_{j}(n-\right.$ 
$\left.\left.k_{i j}\right)\right\}_{n=0}^{\infty}, i=k+1, k+2, \ldots, n$, are nonoscillatory. It follows from (1) that $\left\{\Delta x_{i}(n)\right\}_{n=0}^{\infty}(i=1,2, \ldots, k)$ are oscillatory and

$$
\lim _{n \rightarrow \infty} \Delta x_{i}(n)=0, i=k+1, k+2, \ldots, m .
$$

Hence, for any $\varepsilon>0$, there exist $k$ sequences $\left\{n_{i l}\right\}$ of integers, $i=1,2, \ldots, k$ such that

$$
\left\{\begin{array}{l}
n_{i l} \uparrow \infty,\left|x_{i}\left(n_{i l}\right)\right| \rightarrow U_{i} \text { as } l \rightarrow \infty,\left|x_{i}\left(n_{i l}\right)\right|>U_{i}-\varepsilon, \\
\Delta x_{i}\left(n_{i l}-1\right) \geq 0,\left|x_{i}(n)\right|<U_{i}+\varepsilon \text { for } n \geq n_{1},
\end{array}\right.
$$

for $i=1,2, \ldots, k$, where $n_{1}=\min \left\{n_{i 1}: i=1,2, \ldots, k\right\}$. By going to subsequences if necessary, we may assume $\left|x_{i}\left(n_{i l}\right)\right|=x_{i}\left(n_{i l}\right)$ ( use $-x_{i}(n)$ instead of $x_{i}(n)$ and $-a_{i j}$ instead of $a_{i j}$ for $j \neq i$, if necessary). By (1), as long as $l$ is sufficiently large, we have

$$
0 \leq-\sum_{j=1}^{m} a_{i j} x_{j}\left(n_{i l}-k_{i j}-1\right) \leq-a_{i i} x_{i}\left(n_{i l}-k_{i i}-1\right)+\sum_{j \neq i}^{m}\left|a_{i j}\right|\left(U_{j}+\epsilon\right),
$$

or

$$
x_{i}\left(n_{i l}-k_{i i}-1\right) \leq \frac{1}{a_{i i}} \sum_{j \neq i}^{m}\left|a_{i j}\right|\left(U_{j}+\varepsilon\right), i=1,2, \ldots, k .
$$

Set

$$
\beta_{i l}=\frac{1}{a_{i i}} \sum_{j \neq i}^{m}\left|a_{i j}\right|\left(U_{j}+\varepsilon\right), i=1,2, \ldots, k .
$$

We will now show

$$
\begin{aligned}
& a_{i i} x_{i}\left(n_{i l}\right)+\sum_{j \neq i}^{m} \tilde{a}_{i j}\left(U_{j}+\varepsilon\right) \\
& \quad \leq \frac{2 \varepsilon\left(k_{i i}+1\right) a_{i i}\left[\left(k_{i i}+2\right)\left(3 k_{i i}+4\right)+2 k_{i i}\left(k_{i i}+1\right)^{2} a_{i i}\right]}{\left(3 k_{i i}+4\right)^{2}-\left(k_{i i}+1\right) a_{i i}\left[\left(k_{i i}+2\right)\left(3 k_{i i}+4\right)+2 k_{i i}\left(k_{i i}+1\right)^{2} a_{i i}\right]},
\end{aligned}
$$

for $i=1,2, \ldots, k$. If $x_{i}\left(n_{i l}\right) \leq \beta_{i l}$, then (23) obviously holds. If $x_{i}\left(n_{i l}\right)>\beta_{i l}$, then by (21) and (22) there exists an integer $l_{i}^{*}$ with $0 \leq l_{i}^{*} \leq k_{i i}$ such that

$$
x_{i}\left(n_{i l}-l_{i}^{*}-1\right) \leq \beta_{i l} \text { and } x_{i}\left(n_{i l}-l_{i}^{*}\right)>\beta_{i l} .
$$

Let $\xi_{i l} \in(0,1]$ such that

$$
\begin{aligned}
x_{i}\left(n_{i l}\right. & \left.-l_{i}^{*}\right)-\xi_{i l}\left[x_{i}\left(n_{i l}-l_{i}^{*}\right)-x_{i}\left(n_{i l}-l_{i}^{*}-1\right)\right] \\
& =x_{i}\left(n_{i l}-l_{i}^{*}-1\right)+\left(1-\xi_{i l}\right)\left[x_{i}\left(n_{i l}-l_{i}^{*}\right)-x_{i}\left(n_{i l}-l_{i}^{*}-1\right)\right] \\
& =\beta_{i l} .
\end{aligned}
$$

From (1) we have

$$
\Delta x_{i}(n) \leq a_{i i}\left[-x_{i}\left(n-k_{i i}\right)+\beta_{i l}\right] \leq a_{i i}\left(\left(U_{i}+\varepsilon\right)+\beta_{i l}\right), n_{1} \leq n \leq n_{i l} .
$$


For $n_{i l}-l_{i}^{*}-1 \leq n \leq n_{i l}-1$, summing (25) and using (24), we have

$$
\begin{aligned}
\beta_{i l}-x_{i}\left(n-k_{i i}\right) & \leq \sum_{j=n-k_{i i}}^{n_{i l}-l_{i}^{*}-2} \Delta x_{i}(j)+\left(1-\xi_{i l}\right) \Delta x_{i}\left(n_{i l}-l_{i}^{*}-1\right) \\
& \leq a_{i i}\left(\left(U_{i}+\varepsilon\right)+\beta_{i l}\right)\left(n_{i l}+k_{i i}-l_{i}^{*}-\xi_{i l}-n\right),
\end{aligned}
$$

for $n_{i l}-l_{i}^{*}-1 \leq n \leq n_{i l}-1$. Substituting this into the first inequality in (25), we obtain

$\Delta x_{i}(n) \leq a_{i i}^{2}\left[\left(U_{i}+\varepsilon\right)+\beta_{i l}\right]\left(n_{i l}+k_{i i}-l_{i}^{*}-\xi_{i l}-n\right), n_{i l}-l_{i}^{*}-1 \leq n \leq n_{i l}-1$.

Combining this and (25), we have

$$
\Delta x_{i}(n) \leq a_{i i}\left(\left(U_{i}+\varepsilon\right)+\beta_{i l}\right) \min \left\{1, a_{i i}\left(n_{i l}+k_{i i}-l_{i}^{*}-\xi_{i l}-n\right)\right\},
$$

for $n_{i l}-l_{i}^{*}-1 \leq n \leq n_{i l}-1$. We consider the following two subcases:

SubCase $1 . l_{i}^{*}+\xi_{i l} \leq 2\left(k_{i i}+1\right)^{2} /\left(3 k_{i i}+4\right)$. In this case, by (26) and (7), we have

$$
\begin{aligned}
& x_{i}\left(n_{i l}\right)-\beta_{i l} \\
& =\sum_{n=n_{i l}-l_{i}^{*}}^{n_{i l}-1} \Delta x(n)+\xi_{i l} \Delta x\left(n_{i l}-l_{i}^{*}-1\right) \\
& \leq a_{i i}^{2}\left(\left(U_{i}+\varepsilon\right)+\beta_{i l}\right)\left[\sum_{n=n_{i l}-l_{i}^{*}}^{n_{i l}-1}\left(n_{i l}+k_{i i}-l_{i}^{*}-\xi_{i l}-n\right)+\xi_{i l}\left(k_{i i}+1-\xi_{i l}\right)\right] \\
& =a_{i i}^{2}\left(\left(U_{i}+\varepsilon\right)+\beta_{i l}\right)\left[\left(k_{i i}+1\right)\left(l_{i}^{*}+\xi_{i l}\right)-\frac{1}{2}\left(l_{i}^{*}+\xi_{i l}\right)^{2}-\frac{1}{2}\left(l_{i}^{*}+\xi_{i l}^{2}\right)\right] \\
& \leq a_{i i}^{2}\left(\left(U_{i}+\varepsilon\right)+\beta_{i l}\right)\left[\left(k_{i i}+1\right)\left(l_{i}^{*}+\xi_{i l}\right)-\frac{k_{i i}+2}{2\left(k_{i i}+1\right)}\left(l_{i}^{*}+\xi_{i l}\right)^{2}\right] \\
& \leq \frac{4\left(k_{i i}+1\right)^{4}}{\left(3 k_{i i}+4\right)^{2}} a_{i i}^{2}\left(\left(U_{i}+\varepsilon\right)+\beta_{i l}\right) \\
& \leq \frac{\left(k_{i i}+1\right)}{\left(3 k_{i i}+4\right)^{2}} a_{i i}\left[\left(k_{i i}+2\right)\left(3 k_{i i}+4\right)+2 k_{i i}\left(k_{i i}+1\right)^{2} a_{i i}\right]\left(\left(U_{i}+\varepsilon\right)+\beta_{i l}\right) \\
& \leq \frac{\left(k_{i i}+1\right)}{\left(3 k_{i i}+4\right)^{2}} a_{i i}\left[\left(k_{i i}+2\right)\left(3 k_{i i}+4\right)+2 k_{i i}\left(k_{i i}+1\right)^{2} a_{i i}\right]\left(x_{i}\left(n_{i l}\right)+\beta_{i l}+2 \varepsilon\right) .
\end{aligned}
$$

SuBCASE $2 . l_{i}^{*}+\xi_{i l}>2\left(k_{i i}+1\right)^{2} /\left(3 k_{i i}+4\right)$. In this case, there exists an integer $m_{i}^{*}$ and an $\eta_{i l} \in[0,1)$ such that

$$
m_{i}^{*}+\eta_{i l}=\frac{2\left(k_{i i}+1\right)^{2}}{3 k_{i i}+4} .
$$


Consequently, from (26) we conclude that

$$
\begin{aligned}
& x_{i}\left(n_{i l}\right)-\beta_{i l} \\
&=\xi_{i l} \Delta x_{i}\left(n_{i l}-l_{i}^{*}-1\right)+\sum_{n=n_{i l}-l^{*}}^{n_{i l}-m_{i}^{*}-2} \Delta x_{i}(n)+\left(1-\eta_{i l}\right) \Delta x_{i}\left(n_{i l}-m_{i}^{*}-1\right) \\
& \quad+\eta_{i l} \Delta x_{i}\left(n_{i l}-m_{i}^{*}-1\right)+\sum_{n=n_{i l}-m_{i}^{*}}^{n_{i l}} \Delta x_{i}(n) \\
& \leq a_{i i}\left(\left(U_{i}+\varepsilon\right)+\beta_{i l}\right)\left[\left(\xi_{i l}+l_{i}^{*}-m^{*}-\eta_{i l}\right)+\eta_{i l} a_{i i}\left(k_{i i}+m_{i}^{*}+1-l_{i}^{*}-\xi_{i l}\right)\right. \\
&\left.\quad+a_{i i} \sum_{n=n_{i l}-m_{i}^{*}}^{n_{i l}-1}\left(n_{i l}+k_{i i}-l_{i}^{*}-\xi_{i l}-n\right)\right] \\
&=a_{i i}\left(\left(U_{i}+\varepsilon\right)+\beta_{i l}\right)\left\{\left(\xi_{i l}+l_{i}^{*}\right)\left[1-a_{i i}\left(m_{i}^{*}+\eta_{i l}\right)\right]\right. \\
&\left.\quad+\left[a_{i i}\left(k_{i i}+1\right)-1\right]\left(m_{i}^{*}+\eta_{i l}\right)+\frac{1}{2} a_{i i}\left(m_{i}^{*}+\eta_{i l}\right)^{2}-\frac{1}{2} a_{i i}\left(m_{i}^{*}+\eta_{i l}^{2}\right)\right\} \\
& \leq a_{i i}\left(\left(U_{i}+\varepsilon\right)+\beta_{i l}\right)\left\{\left(\xi_{i l}+l_{i}^{*}\right)\left[1-a_{i i}\left(m_{i}^{*}+\eta_{i l}\right)\right]\right. \\
&\left.+\left[a_{i i}\left(k_{i i}+1\right)-1\right]\left(m_{i}^{*}+\eta_{i l}\right)+\frac{k_{i i}}{2\left(k_{i i}+1\right)} a_{i i}\left(m_{i}^{*}+\eta_{i l}\right)^{2}\right\} \\
& \leq a_{i i}\left(\left(U_{i}+\varepsilon\right)+\beta_{i l}\right)\left[k_{i i}+1-\left(m_{i}^{*}+\eta_{i l}\right)+\frac{k_{i i}}{2\left(k_{i i}+1\right)} a_{i i}\left(m_{i}^{*}+\eta_{i l}\right)^{2}\right] \\
&= \frac{\left(k_{i i}+1\right)}{\left(3 k_{i i}+4\right)^{2}} a_{i i}\left[\left(k_{i i}+2\right)\left(3 k_{i i}+4\right)+2 k_{i i}\left(k_{i i}+1\right)^{2} a_{i i}\right]\left(\left(U_{i}+\varepsilon\right)+\beta_{i l}\right) \\
& \leq \frac{\left(k_{i i}+1\right)}{\left(3 k_{i i}+4\right)^{2}} a_{i i}\left[\left(k_{i i}+2\right)\left(3 k_{i i}+4\right)+2 k_{i i}\left(k_{i i}+1\right)^{2} a_{i i}\right]\left(\left(x_{i}\left(n_{i l}\right)+\beta_{i l}+2 \varepsilon\right) .\right.
\end{aligned}
$$

Combining Cases 1 and 2, we have

$$
\begin{aligned}
& a_{i i} x_{i}\left(n_{i l}\right) \\
& \leq \frac{\left(3 k_{i i}+4\right)^{2}+\left(k_{i i}+1\right) a_{i i}\left[\left(k_{i i}+2\right)\left(3 k_{i i}+4\right)+2 k_{i i}\left(k_{i i}+1\right)^{2} a_{i i}\right]}{\left.3 k_{i i}+4\right)^{2}-\left(k_{i i}+1\right) a_{i i}\left[\left(k_{i i}+2\right)\left(3 k_{i i}+4\right)+2 k_{i i}\left(k_{i i}+1\right)^{2} a_{i i}\right]} \\
& \quad \times \sum_{j \neq i}^{m}\left|a_{i j}\right|\left(U_{j}+\epsilon\right) \\
& \quad+\frac{2 \epsilon\left(k_{i i}+1\right) a_{i i}\left[\left(k_{i i}+2\right)\left(3 k_{i i}+4\right)+2 k_{i i}\left(k_{i i}+1\right)^{2} a_{i i}\right]}{\left(3 k_{i i}+4\right)^{2}-\left(k_{i i}+1\right) a_{i i}\left[\left(k_{i i}+2\right)\left(3 k_{i i}+4\right)+2 k_{i i}\left(k_{i i}+1\right)^{2} a_{i i}\right]}
\end{aligned}
$$


for $i=1,2, \ldots, k$. This implies (23) is true. Let $l \rightarrow \infty$ and $\varepsilon \rightarrow 0$ in (23), we obtain

$$
a_{i i} U_{i}+\sum_{j \neq i}^{m} \tilde{a}_{i j} U_{j} \leq 0, \quad i=1,2, \ldots, k .
$$

On the other hand, for each $i=k+1, \ldots, m$, let $\left\{s_{i l}\right\}_{l=1}^{\infty} \uparrow \infty$ be sequence of integers such that $\lim _{l \rightarrow \infty} x_{i}\left(s_{i l}\right)=U_{i}$. By (19), we have $\lim _{l \rightarrow \infty} \Delta x_{i}\left(s_{i l}+\right.$ $\left.k_{i i}\right)=0$. Using (1) we have

$$
\begin{aligned}
0 & =\Delta x_{i}\left(s_{i l}+k_{i i}\right)+a_{i i} x_{i}\left(s_{i l}\right)+\sum_{j \neq i}^{m} a_{i j} x_{j}\left(s_{i l}+k_{i i}-k_{i j}\right) \\
& \geq \Delta x_{i}\left(s_{i l}+k_{i i}\right)+a_{i i} x_{i}\left(s_{i l}\right)+\sum_{j \neq i}^{m} \tilde{a}_{i j}\left|x_{j}\left(s_{i l}+k_{i i}-k_{i j}\right)\right|,
\end{aligned}
$$

since $\tilde{a}_{i j} \leq-\left|a_{i j}\right| \leq 0$. Letting $l \rightarrow \infty$, we obtain

$$
a_{i i} U_{i}+\sum_{j \neq i}^{m} \tilde{a}_{i j} U_{j} \leq 0, i=k+1, k+2, \ldots, m .
$$

By (27) and (28) and using the fact that $\tilde{A}$ is an $M$-matrix (so that $\tilde{A}^{-1}$ is a positive matrix), we have $U_{1}=U_{2}=\cdots=U_{m}=0$. The proof is complete.

\section{Discussion}

Applying Theorem 2.1 to equation (3), we have the following statement.

Corollary 3.1. Assume that

$$
0<a, d<\frac{3}{2(k+1)}+\frac{1}{2(k+1)^{2}}
$$

and

$$
\begin{aligned}
a d> & \frac{(3 k+4)^{2}+(k+1) a\left[(k+2)(3 k+4)+2 k(k+1)^{2} a\right]}{(3 k+4)^{2}-(k+1) a\left[(k+2)(3 k+4)+2 k(k+1)^{2} a\right]} \\
& \times \frac{(3 k+4)^{2}+(k+1) d\left[(k+2)(3 k+4)+2 k(k+1)^{2} d\right]}{(3 k+4)^{2}-(k+1) d\left[(k+2)(3 k+4)+2 k(k+1)^{2} d\right]}|b c| .
\end{aligned}
$$

Then every solution $\left(x_{1}(n), x_{2}(n)\right)$ of (3) tends to 0 as $n \rightarrow \infty$.

Obviously, when $c=0$ or $b=0,(30)$ holds naturally. In view of Corollary $3.1,(29)$ is the sufficient condition for asymptotic stability of (3). Note that

$$
2 \cos \frac{k \pi}{2 k+1}=2 \sin \frac{\pi}{2(2 k+1)} \approx \frac{3}{2} \frac{1}{(k+1)}+\frac{1}{2} \frac{1}{(k+1)^{2}} .
$$

This shows the conditions for asymptotic stability in Theorem 2.1 are rather careful. 


\section{REFERENCES}

[1] H. Matsunaga and T. Hara, The asymptotic stability of a two-dimensional linear delay difference equation, Dynamics of Cont. Discrete Impulsive Sys. 6 (1999), 465-473.

[2] J. W. Wu and K. S. Hong, Delay-independent exponential stability criteria for time varying discrete delay systesm, IEEE Trans. Automatic Control 39 (1994), 811-814.

[3] M. Fiedler, Special Matrices and Their Applications in Numerical Mathematics, Martinus Nijhoff Publ. (Kluwer), Dordrecht, 1986.

[4] B. Bapat and T. E. S. Raghavan, Nonnegative Matrices and Applications, Cambridge University Press, Cambridge, 1997.

X.H. Tang

Department of Applied Mathematics

Central South University

Changsha, Hunan 410083, P.R. China

E-mail, X.H. Tang: tangxh@mail.csu.edu.cn

S.S. Cheng

Department of Mathematics

Tsing Hua University

Hsinchu 30043, Taiwan, ROC

Received: 17.12.2003.

Revised: 15.3.2004. 